\title{
DESARROLLO (ECONÓMICO) LOCAL Y GLOBALIZACIÓN ${ }^{1}$
}

\section{LOCAL ECONOMIC DEVELOPMENT AND GLOBALIZATION}

\author{
César Vallejo Mejía \\ Rector
}

Universidad Autónoma de Manizales
Palabras Clave: Desarrollo, economía, globalización, sociedad, bienestar, políticas, estrategias, local

Key words: Development, economy, globalization, society, wel fare, policies, strategies, local.

\section{I - INTRODUCTION}

In order to diminish the risks (threats) and to profit from the opportunities associated with globalization in the context of local economic development, it is necessary to strengthen attitudes towards what is "local" and in conjunction therewith, to develop objectives and design and apply strategies and policies.

In support of the foregoing thesis, the following pages will develop and clarify the concept of local economic development used for purposes of analysis, as well as well as the manner in which the concept of globalization is perceived, its origins, the forces that drive it and its potential impact (risks and opportunities) on economic, political and cultural developmental aspects. The presentation concludes by demonstrating the importance of strengthening attitudes towards the concept of the "local" as well as of formulating and implementing strategies and policies in terms of locally centered and developed

1 Ponencia presentada en el Seminario Internacional "Desarrollo Económico Local" Bogotá, Julio 25, 26 y 27 de 2007

2 Profesional en Filosofía y Letras. Maestría en Economía. Doctorado en Economía en Christian Albrecht Universitat. Doctorado en Ciencias Sociales con énfasis en Niñez y Juventud. Ex director del Departamento Nacional de Planeación. Rector de la Universidad Autónoma de Manizales UAM.

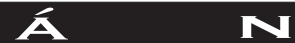

Universidad Autónoma de Manizales

\section{$\mathbf{R}$}

Año I4, Número 23, julio - diciembre 2007 
objectives ("autochthonous development) in order to successfully overcome the challenge posed by globalization to local development. (The local as key to success of the global).

\section{I - INTRODUCCIÓN.}

Para atenuar los riesgos (amenazas) y aprovechar las oportunidades asociadas con la Globalización, en relación con el Desarrollo Económico Local, es necesario profundizar el sentido de lo "local" y, en función de él, formular objetivos, diseñar y aplicar estrategias y políticas.

Con el fin de sustentar la tesis anterior, en las páginas siguientes se explicita el concepto de Desarrollo Económico Local, que se utiliza en el análisis, así como la forma como se entiende la globalización, sus "fuerzas y determinantes", y su posible impacto (riesgos y oportunidades) sobre las dimensiones económica, política y cultural del desarrollo. La presentación concluye mostrando la importancia de profundizar el concepto de lo local y de formular y aplicar estrategias y políticas en función de objetivos "propios" ("desarrollo propio"), para sortear exitosamente el desafío que representa la globalización (amenazas y oportunidades) sobre el Desarrollo Local. (Lo Local como clave del éxito en lo Global).

\section{II - DESARROLLO ECONÓMICO LOCAL.}

Se puede definir Desarrollo como el cumplimiento, por parte de una Sociedad, (el grado de logro), de los objetivos y metas que se ha propuesto.

En la anterior definición se deben resaltar tres elementos importantes: la Sociedad como sujeto del Desarrollo, los objetivos y metas que se propone, y la forma (modelos, políticas y estrategias) que adopta y aplica para conseguirlos.

\section{1 - La Sociedad, Sujeto (Autor y Beneficiario) del Desarrollo.}

Desarrollo es el nombre que adquiere el progreso cuando se refiere a la especie humana. Cuando la evolución, después de una búsqueda ciega y milenaria, logra crear el espíritu y aparece el ser humano (la naturaleza alcanza autonomía), da lugar al Desarrollo (evolución o 
progreso con Sujeto). La fuerza preeminente en su conducción ya no es el azar sino la decisión humana (ilustrada por la inteligencia y el conocimiento) que, en colectivo, busca su progreso y bienestar creciente.

El sujeto del Desarrollo es el ser humano como Sociedad, es decir, como colectivo organizado, en el que los individuos, con su potencial e intereses específicos, articulan sus diferencias y adoptan unas reglas de juego que les permiten resolver más fácilmente problemas comunes y converger en metas y objetivos de progreso.

Sin Sociedad no tiene sentido hablar de Desarrollo. Formar Sociedad (con todo lo que ello implica) es, por eso, la primera y fundamental tarea del Desarrollo, (dimensión política), porque su conformación no es el resultado de un proceso automático ni estándar (Habermas). Hay tantas formas de construir sociedad como "localidades" o pueblos con potencial, problemas, intereses, metas y objetivos que difieren, en cuanto condicionados por culturas, historias y geografías distintas.

La Sociedad como un todo, todos sus integrantes, es autora y beneficiaria del Desarrollo. Como referido a la Sociedad, el Desarrollo es por esencia incluyente. La formulación de sus objetivos, su construcción y logro, así como la participación en sus beneficios deben comprometer a todos los integrantes de la sociedad. Sin vulnerar uno de los derechos fundamentales de las personas, el respeto por su autonomía, uno de los objetivos mínimos de la Sociedad debe ser el de garantizarle a todos sus integrantes las condiciones para que puedan aportar, cada uno desde su potencial específico, en la construcción del Desarrollo y participar en sus beneficios. Por ello son tan importantes los indicadores de inclusión, al lado de los indicadores propios de las distintas dimensiones del Desarrollo. No es avanzada una Sociedad, en la medida en que sus integrantes no tengan acceso a esas condiciones. Con la misma lógica, el atraso en las localidades de una Región, o en las regiones de una Nación, son síntoma de atraso en aquélla y ésta, respectivamente.

\section{2 - Los Objetivos del Desarrollo: la Función de Bienestar.}

La fuerza que cohesiona (que forma) la Sociedad y articula las diferencias entre los individuos es la convergencia en objetivos que de

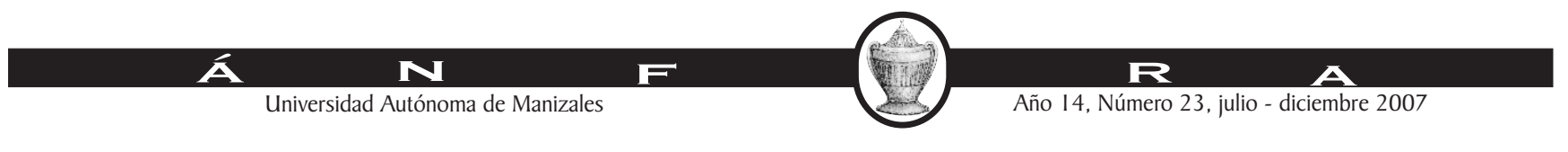


alguna manera comparten. Esta convergencia es más o menos fuerte, más o menos generadora de progreso y muestra un mayor o menor nivel de Desarrollo, en la medida en que trasciende la solución de necesidades básicas y la satisfacción de "derechos" fundamentales de las personas (objetivos "mínimos", comunes a todas las sociedades), y se propone el logro de objetivos específicos, difícilmente repetibles porque están asociados al potencial particular de los individuos que la integran, a la historia, la cultura y la geografía que le son propias (objetivos "máximos", podemos llamarlos, a la manera como se habla en ética de mínimos y máximos).

La formulación, explícita o implícita, de los objetivos de progreso de una Sociedad, es lo que los economistas denominan "Función de Bienestar". Toda Sociedad se cohesiona, entonces, alrededor de una Función de Bienestar que le es propia, cuya "maximización" es la tarea del Desarrollo (el logro más eficiente posible de los objetivos que la conforman).

Los objetivos que integran el argumento de la Función de Bienestar de una Sociedad, se pueden clasificar en Objetivos Mínimos y Objetivos Máximos. En ambos casos la Sociedad reconoce la satisfacción, en el presente y en el futuro, de intereses estrictamente individuales (más cercanos a la dimensión social del desarrollo) y de intereses colectivos (dimensiones económica, política e institucional).

Los Objetivos Mínimos de toda sociedad, en relación con los individuos, están relacionados con las necesidades básicas y los derechos fundamentales de las personas. De ellos forman parte niveles mínimos de salud, nutrición, educación y vivienda, así como el derecho fundamental a la vida, la libertad y la "inclusión" social que involucra, no solo la posibilidad de participar en los beneficios del progreso, sino la capacidad de aportar en su construcción (ésta como condición de aquélla). Allí se incluyen, por lo tanto, las competencias básicas y las competencias ciudadanas, necesarias para interactuar con los demás, la formación para el trabajo y todo lo que haga posible la capacidad de "agencia" o el "Desarrollo como Libertad", como lo llama Amartya Sen.

En relación con el colectivo, los Objetivos Mínimos de toda Sociedad representan el derecho a la autodeterminación de los pueblos, de fijarse

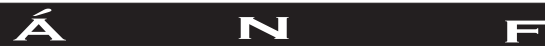

Universidad Autónoma de Manizales 
sus Metas y Objetivos Máximos y de elegir a sus gobernantes; el derecho a la Sostenibilidad o aprovechamiento económico de los recursos naturales sin atentar contra la posibilidad de sobrevivencia por parte de las generaciones futuras, y la posibilidad de adoptar las "instituciones" o reglas de juego necesarias para hacer eficiente y armónica la interacción con los demás. Allí se incluye el derecho a la equidad o la garantía de acceso a los beneficios y apoyos que ofrece la Sociedad, por parte de todos sus integrantes.

En cuanto a los Objetivos Máximos (locales o "propios"), la Función de Bienestar incluye intereses y características que distinguen a la Sociedad de las demás y que, para ella, representan progreso y mayor bienestar: el despliegue del potencial peculiar de los individuos que la integran; el fortalecimiento de su Racionalidad colectiva o forma propia de entender el mundo, en cuanto determinada por su Cultura, su Historia y su Geografía; la construcción de su economía con base en los recursos naturales que le son propios.

La importancia de la anterior clasificación radica en que los Objetivos Mínimos de una Sociedad, al ser comunes a otras sociedades, pueden ser "globalizables" (objeto de políticas comunes y de indicadores que comparan el desarrollo entre países, en el logro de esos objetivos específicos). Los Objetivos Máximos, por el contrario, no son globalizables porque son "propios" o específicos de una Sociedad particular (son el componente estrictamente "local" de la Sociedad ${ }^{3}$ ) y, por eso mismo, cuando la Sociedad los fortalece y defiende contra la estandarización, son la mejor defensa contra la competencia global y el mejor recurso para tener éxito en un mercado internacional ampliado (lo "local" como factor de éxito en lo "global").

Desarrollo es pues un fenómeno integral, que representa la búsqueda de los Objetivos Mínimos y Máximos de una Sociedad y solo tiene sentido como referido a ella. Es "Propio" o "Local" ${ }^{\text {y }}$ y complejo como ella; se refiere al conjunto de actuaciones mediante las cuales una sociedad progresa en busca de sus objetivos. Incluye, por tanto, las dimensiones interdependientes, económica, social, política e institucional, en las que se manifiesta la "vida" de una Sociedad (su desarrollo) y entre las cuales, las teorías que tratan de explicar el Desarrollo establecen diversos nexos de causalidad. La expresión "desarrollo económico" se
3 El Desarrollo es por esencia local. Desde el ámbito local se puede pasar a escenarios de mayor alcance como la región o la nación, pero no al revés. En ese sentido no es posible hablar de países desarrollados donde existan regiones o localidades que no lo sean.

4 Lo local, definido como escenario de "vida" (de desarrollo) de una sociedad concreta, se puede concebir como la célula territorial de la Sociedad, el ámbito desde el cual puede extenderse a lo regional, a lo nacional y aun a lo mundial, a medida que renuncia a características específicas $y$, en ese sentido, empobrece o "minimiza" sus objetivos (los reduce a lo que es común a cada una de esas instancias).

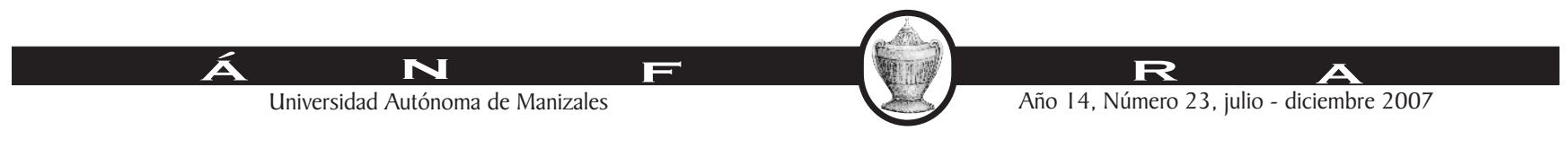


refiere, por tanto, a una de las dimensiones de la dinámica social (la mirada del desarrollo desde la perspectiva económica). De igual manera se pueden utilizar expresiones como "desarrollo social", "desarrollo político", o "desarrollo institucional". ${ }^{5}$

No tiene sentido, por lo tanto, hablar de desarrollo económico, social o político, como si fueran fenómenos independientes. ${ }^{6}$ En realidad se trata de dimensiones necesarias, pero ninguna de ellas suficiente. Los indicadores que les son propios muestran aspectos de un único fenómeno, el Desarrollo, en el cual se está más o menos avanzado, en relación a las Metas y Objetivos formulados por cada Sociedad.

Las medidas estándar del Desarrollo (aplicables a todas las sociedades por igual) y las comparaciones que con base en ellas se hacen, solo pueden utilizarse en forma restringida. Si el mayor o menor nivel de desarrollo se determina en relación con el grado de cumplimiento de las metas y objetivos de una Sociedad concreta, las comparaciones entre sociedades solo pueden establecerse en la medida en que esos objetivos incluyan metas comunes a otras sociedades (Objetivos Mínimos). Es el caso de las Metas del Milenio adoptadas por distintas sociedades por iniciativa de la Organización de Naciones Unidas. En este caso se trata de indicadores parciales sobre objetivos mínimos reconocidos por esas sociedades. En relación con objetivos máximos, propios de cada sociedad, no tiene mucho sentido establecer comparaciones (entre países o regiones). El peligro de la "clasificación" que ordena las sociedades entre desarrolladas y no desarrolladas, tan frecuentes en estadísticas sobre el desarrollo, es que le restan importancia a los Objetivos Máximos de la función de Bienestar y desconocen que el Desarrollo, como el Progreso, no tiene metas finales.

\section{3 - Políticas y estrategias: el proceso de Maximización.}

Establecida la Función de Bienestar, o los Objetivos que una Sociedad se propone, su tarea es alcanzarlos de la manera más eficiente posible. Es lo que los economistas denominan "maximización" u "optimización" de la Función de Bienestar.7 Para ello dispone de recursos escasos, propios o ajenos, y de teorías que sistematizan experiencias parecidas.

Como en el caso de los Objetivos de Desarrollo (argumento de la Función de Bienestar), también en su proceso de maximización, en las
5 En el caso de la dimension económica, son cada vez más convincentes las teorías que muestran el papel preponderante (aunque no suficiente) que tienen las otras dimensiones sobre el "desarrollo económico": desarrollo social y capital humano (Gary Becker), desarrollo institucional (North Douglas) y desarrollo político (John Rawls, Robert Puttnam).

6 Una sociedad puede mostrar indicadores económicos muy positivos sin que ello signifique niveles altos de desarrollo; o políticos o sociales.

7 Partiendo de sus supuestos sobre la conducta racional de las personas y de las herramientas propias de su disciplina, los Economistas formulan la Función de Bienestar Social en términos del consumo y el aporte de factores de producción por parte de los agentes económicos que conforman una Sociedad. Sujeta al criterio Paretiano, la Sociedad maximiza su Bienestar, con mayor o menor intervención del Estado, según la situación de partida, mediante la actividad de sus integrantes que, con el conocimiento y la información de que disponen (con las condiciones que les garantiza la misma sociedad), buscan el mayor provecho en su consumo o en su actividad productiva, sujetos a las restricciones impuestas por la escasez, la distribución de recursos y la disponibilidad tecnológica. Los objetivos de los individuos, que determinan su intervención en la Sociedad, incluyen de alguna manera ( $y$ ello es indicador de desarrollo) los objetivos mínimos y máximos de que se habla en el texto.

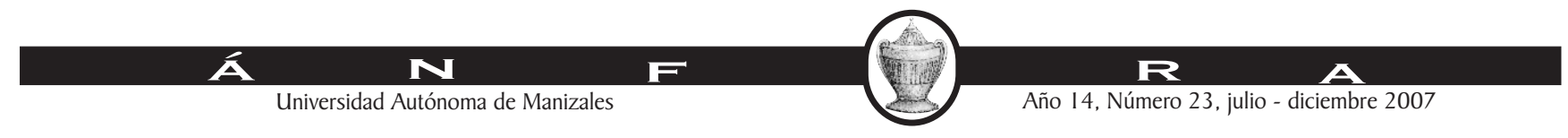


políticas y estrategias que utiliza la Sociedad para conseguirlos, hay aspectos "globalizables", y aspectos no globalizables que hay que defender ante la presión estandarizante de la globalización.

Los primeros, teorías y modelos, comunes a cualquier Sociedad, forman parte del estado del arte sobre gestión del desarrollo y, en ese sentido, son aplicables en cualquier sociedad (estandarizables). El papel determinante en el crecimiento económico, de la inversión (con recursos propios o ajenos), la tecnología y la sinergia entre las distintas formas de capital; la importancia de la formación de capital humano, de instituciones o reglas de juego claras y eficaces y del capital social como determinantes de la calidad en las decisiones que toman los individuos, las empresas y las organizaciones de la Sociedad (condiciones de eficiencia social); el papel reconocido de la ciencia, la tecnología y la innovación permanente; la función multiplicadora de las cadenas productivas y los clusters de apoyo, así como de las redes y sistemas de innovación, y del acceso a los avances crecientes en conectividad y al mundo digital; las ventajas insospechadas que ofrece la llamada Sociedad del Conocimiento y su potencial en la formación de agentes creativos (individuos o grupos) y en la construcción de lo que se ha dado en llamar "Regiones o sociedades inteligentes".... Estas y otras muchas estrategias y políticas de planeación y desarrollo, documentadas en investigaciones empíricas, difundidas en Universidades y consagradas en protocolos de las Agencias para el Desarrollo, forman la extensa lista de lo globalizable en materia de gestión y maximización de las Funciones de Bienestar.

Lo no globalizable, en la obtención eficiente de los Objetivos "propios" (mínimos y máximos), es la aplicación concreta de las estrategias y recomendaciones globalizadas a la "situación" específica de la Sociedad; las políticas deben ajustarse a las características espaciotemporales del desarrollo "local" y a sus condicionamientos históricos, culturales y geográficos. La capacidad de confrontar las teorías con la realidad, y de crear fórmulas e instituciones apropiadas (que incluyen la opción política sobre la forma y el alcance de la intervención del estado), frecuentemente en contra de las presiones estandarizantes de la globalización, es determinante en la maximización de la Función de Bienestar. 


\section{III - GLOBALIZACIÓN Y DESARROLLO LOCAL.}

La revolución de las comunicaciones, caracterizada por la magnitud de sus cambios tecnológicos, su innovación permanente y la rapidez de su difusión, ha densificado el mundo, minimizando las distancias y haciendo más próximos los pueblos, las sociedades y sus integrantes. En los términos de Thomas Friedman, la tierra se ha hecho plana y la globalización, que en el siglo XIX había llegado a las naciones y en el siglo XX a las empresas, ahora, en el siglo de la accesibilidad y la conectividad, ha llegado a las personas.

Desaparecen las fronteras y se relativiza la dimensión espacio-temporal en la interacción entre los pueblos, se difunden las ideas, se divulgan las políticas, se mueven las personas, se contagian las culturas, se acortan al máximo las ventajas tecnológicas, se dinamizan las alianzas como forma de creación de valor y de producción más eficiente, se internacionalizan los mercados de bienes y servicios, fluyen sin pausa los capitales en busca de las mayores rentabilidades. Es la nueva forma de la globalización, producida por la revolución de las comunicaciones.

Cabalgando sobre una enorme ola de progreso tecnológico, que no parece tener fin, el complejo fenómeno de la globalización ha penetrado todas las dimensiones de la vida en sociedad y ha generado profundas dinámicas de cambio, sin que nadie lo planificara o previera en todas sus consecuencias, oportunidades y riesgos. "Una nueva estructura social dominante, la sociedad red; una nueva economía, la economía informacional/global; y una nueva cultura, la cultura de la virtualidad real", en palabras de Manuel Castells. ${ }^{8}$

No se pueden menos que reconocer las grandes oportunidades que ofrece y hacen atractiva la globalización para el Desarrollo Local, en todas sus dimensiones.

- La internacionalización o ampliación completa de los mercados, en los que también lo "propio" tiene la oportunidad de ser valorado y potencializado como palanca del desarrollo local.

- El acceso a un capital cada vez mayor, que no tiene nacionalidad, que está a la caza de buenas oportunidades, y que se puede poner al servicio de negocios basados en ideas y recursos locales.
8 Manuel Castells, "La era de la información: economía, sociedad y cultura", Conclusión, vol.III, Siglo XXI editores, México, 1997. 
- La disponibilidad de información y tecnología, característica de la Sociedad del Conocimiento, que complementa los esfuerzos locales en Investigación, Ciencia e Innovación o suple sus deficiencias.

- La oportunidad de hacer alianzas y emprender procesos de integración vertical y horizontal, que dinamizan la investigación científica, la innovación y la producción locales.

- La cooperación internacional en programas de desarrollo, por parte de países avanzados y de Instituciones de carácter global, como la OMC, la ONU, el FMI y la Banca Multilateral.

- La difusión y adopción de normas, valores e instituciones compartidas por las distintas sociedades, que impactan benéficamente las dimensiones económica, política y cultural del desarrollo local: la democratización y reivindicación del individuo (de sus derechos y potencialidades); los derechos fundamentales de personas y sociedades; un nuevo sistema de relaciones entre las personas y de éstas con las organizaciones y con la naturaleza, basado en el respeto ${ }^{9}$ por las diferencias, que delimita y reivindica a la vez, los derechos del individuo frente al Estado, armoniza la relación entre lo público y lo privado, entre gobierno y sociedad civil, entre región y nación (la descentralización basada en el derecho y la capacidad de las comunidades locales a ejercer su autonomía; y aplicada en función del desarrollo local).

Pero la globalización también representa riesgos para el Desarrollo Local que es necesario neutralizar. Los dos más importantes tienen que ver con la Asimetría en el punto de partida de las distintas sociedades, particularmente en relación con la dimensión económica del Desarrollo Local, y con el ímpetu estandarizante de la globalización en lo económico, lo político y lo cultural.

La globalización no reconoce asimetrías en los niveles de desarrollo de las sociedades, en la capacidad real y actual que tienen de acceder a las oportunidades que ella representa o de defenderse de sus amenazas y, por eso mismo, puede convertirse en factor de empobrecimiento de las
9 La globalización nos ha ayudado a entender que "la historia de la humanidad es la historia de sus relaciones con la naturaleza" dice $\mathrm{R}$. Guimaräes. 
que están en desventaja y de ampliación de las diferencias entre países, entre sectores de población y entre regiones al interior de ellos.

En la dimensión económica, por ejemplo, es claro que la globalización y las fuerzas del mercado no generan mecanismos de control que defiendan las estructuras económicas locales de una competencia desigual que las destruye, o regulen el apetito de la inversión extranjera directa que prefiere la especulación (dañina en la mayoría de los casos) a la construcción y fortalecimiento del aparato productivo local (la inversión deja, entonces, de ser un activo productivo y un factor de crecimiento). ${ }^{10}$

La otra amenaza de la Globalización tiene que ver con el atractivo de la estandarización que, sustentada en modelos abstractos y en la experiencia de otros países, parece simplificar la tarea del desarrollo, la maximización de la función de Bienestar, mediante el transplante de instituciones, estrategias y políticas a la realidad local. Se alivia, equivocadamente, la responsabilidad de crear modelos propios, estrategias, instituciones y políticas pertinentes, ajustadas a la realidad local (a sus recursos y geografía, a su historia y su cultura).

Pero quizás la mayor amenaza se da a nivel de la Función de Bienestar de la Sociedad, cuando sus Objetivos Máximos o "propios", sucumben, son olvidados o subestimados, ante el deslumbrante atractivo y la fuerza estandarizante de la Globalización, no pocas veces, bajo la presión de organismos internacionales de cooperación y desarrollo o el influjo sutil de los indicadores que ellos y los académicos utilizan para realizar comparaciones entre países (económicos, sociales, institucionales, tecnológicos) y que, si bien, son adecuados para medir los Objetivos Mínimos del desarrollo, comunes a todos los países, no tienen en cuenta los Máximos locales. (No son por supuesto las Agencias Internacionales las encargadas de defender estos últimos, aunque sí deberían hacerlo los académicos).

Es como si la identidad local (regional y nacional) perdiera su pulso con la "moda" y evadiera el desafío que le plantea su relación con el mundo. Pierde relevancia el poder de las individualidades porque se pone al servicio exclusivo de objetivos "copiados", válidos en otras culturas, o de ramas de la producción que no juegan con los recursos locales. Se
10 Chica Ricardo, "Latinoamérica frente a la globalización: una estrategia alternativa de desarrollo", Fondo Editorial UAM, Manizales, 2007
A N

Universidad Autónoma de Manizales

\section{$\mathbf{R}$}

Año I4, Número 23, julio - diciembre 2007 
desvanecen la historia, la cultura y la geografía como motores del desarrollo local. No solo se empobrece el contenido de la Función de Bienestar, el Desarrollo se hace cada vez menos "propio" y "local", sino que se dificulta la posibilidad de conseguirlo o de maximizarlo (de progresar o avanzar en Desarrollo), porque la amenaza de la Globalización se hace inminente y difícil de neutralizar.

La debilidad de la Función de Bienestar local y su vulnerabilidad ante las fuerzas globalizantes se da, principalmente, cuando no es el resultado de procesos en los que los integrantes de la sociedad articulan sus diferencias y convergen alrededor de propósitos colectivos de largo plazo; cuando la formulación de los objetivos del desarrollo no ejerce el papel cohesionador de la Sociedad y la Función de Bienestar es formulada y adoptada en forma tecnocrática o autoritaria, sin procesos de participación ciudadana que faciliten su apropiación.

\section{IV - RESUMEN Y CONCLUSIÓN.}

1 - A pesar de expresiones frecuentes en la literatura sobre globalización, no tiene sentido hablar de Sociedad Global, como si se tratara de una colectividad "uniformizada" por los efectos de proximidad, conectividad, comunicación inmediata y densificación del mundo, que ha producido la revolución tecnológica de las comunicaciones.

2 - La Sociedad y los individuos que la conforman, tiene una referencia necesaria e insustituible: la historia, la cultura y la geografía propias (específicas e irrepetibles). Por eso la Sociedad siempre es "local" ("situada" como diría Carlos Matus).

3 - El desarrollo, cuyo autor y beneficiario es la Sociedad, es el proceso de cumplimiento (de maximización o de logro eficiente) de los objetivos propios (mínimos y máximos) de esa Sociedad. Por eso, como ella, siempre es "local" (y por analogía, regional y nacional, pero siempre a partir de lo "local").

4 - La globalización abre enormes oportunidades de progreso al desarrollo "local", siempre y cuando no destruya su carácter de "local", es decir, los objetivos "máximos" de su función de bienestar (los

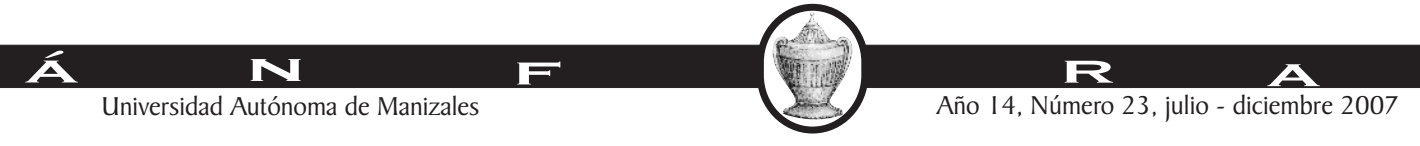


condicionados por la historia, la cultura y la geografía propias de la sociedad), y la forma apropiada de optimizar esa función (de conseguir sus objetivos mínimos y máximos) con la ayuda de teorías y modelos de desarrollo, ajustadas a la "situación" y a los condicionamientos específicos de la sociedad.

5 - La amenaza de la globalización radica en el atractivo simplificador de la estandarización que debilita la identidad local, subestima los objetivos máximos de la sociedad, desvanece la historia, la cultura y la geografía como motores claves del desarrollo local y "transplanta" modelos, estrategias y políticas que no son apropiados para conseguirlo. Cuando no se la neutraliza, acentúa las diferencias y asimetrías en el desarrollo de los pueblos y grupos de población, genera concentración económica y pobreza.

6 - Profundizar y fortalecer el sentido de lo "propio", como expresión del ser histórico, cultural y territorial de la Sociedad, y en función de él formular objetivos que la cohesionen y estrategias que se ajusten a su realidad, es la mejor forma de neutralizar las amenazas de la Globalización, de aprovechar sus oportunidades y de lograr un mayor desarrollo local. 